\title{
Time-dependent changes in inaccessible memory
}

\author{
RUSSELL E. MORGAN \\ Western Illinois University, Macomb, Illinois \\ ROBERT W. FLINT, JR. \\ Mankato State University, Mankato, Minnesota \\ and \\ DAVID C. RICCIO \\ Kent State University, Kent, Ohio
}

\begin{abstract}
The present experiment examined whether forgetting of contextual attributes-the loss of discriminability of background stimulus features over time-also occurs when a memory remains inaccessible (amnestic) during the retention interval. To examine this issue, rats received an acute hypothermia treatment that caused reversible anterograde amnesia for a passive-avoidance task and were tested 1 or 7 days later in either the same or a different context. At the short retention interval, the subjects discriminated between the contexts, as evidenced by the finding that reversal of amnesia occurred only in the training context. However, at the 7-day interval, recovery of the avoidance response was induced in either context, reflecting the forgetting of differentiating characteristics. These findings indicate that inaccessible memory remains susceptible to processes of modification and distortion that typically influence intact memories.
\end{abstract}

Memory is not simply recorded in a permanent unalterable form but can be modified and/or updated through processes of reconstruction or incorporation of new information. For example, a recent series of studies by Loftus and others (Lindsay, 1990; Loftus, 1993; Loftus \& Hoffman, 1989) has dramatically demonstrated this characteristic malleability of memory. Using an eyewitness memory paradigm, these studies have shown that established memories about a previously witnessed event can be modified through the introduction of new, inconsistent information (but see McCloskey \& Zaragoza, 1985, for an alternative interpretation). Apparently, this so-called misinformation effect is just one of many means by which established memories may be distorted or updated over time (see also Belli, 1989; Gordon, McCracken, Dess-Beech, \& Mowrer, 1981; Richardson, Riccio, \& Smoller, 1987).

But do these same phenomena occur when the original memory is inaccessible to retrieval, as in the case of amnesia or repression? That is, are inaccessible memories protected from the influence of subsequent learning, or are they subject to modification and distortion in much the same manner as accessible memories? This issue is not only of theoretical importance for understanding the nature of memory processes but is also of some applied interest, in view of the numerous cases of adults reporting apparent reactivation of early childhood memories concerning trauma and/or abuse (for an interesting discussion

This research was supported in part by NIMH Grant 37535 to D.C.R. Correspondence and reprint requests should be addressed to R. E. Morgan, Psychology Department, 100 Waggoner Hall, Western Illinois University, Macomb, IL 61455 (e-mail: russell_morgan@ccmail.wiu.edu). of issues concerning recovery of unconscious memories, see Erdelyi, 1996). The veridicality of these long-dormant memories is of concern to legal scholars and memory researchers alike. Are these recovered memories accurate, having been "frozen in time," or might they, too, be subject to distortion from various sources, just as are accessible memories?

Although experimental investigation of this question is notoriously difficult, a recent study using animals suggests that a target memory rendered inaccessible (through an amnesia treatment) may nevertheless remain susceptible to modification (Morgan \& Riccio, 1994). In that study, anterograde amnesia (AA) for a fear conditioning episode was induced in rats by training them while in a mildly hypothermic state. One day later, these subjects, now amnesic with respect to the fear episode, received either 0,5 , or $10 \mathrm{~min}$ of innocuous exposure to the conditioning cues. For control subjects, such an extinction treatment weakens fear. But would the exposure have any consequence in animals with amnesia? To assess any such effect, recovery from amnesia was induced by reexposing subjects to the amnestic treatment (hypothermia) shortly before testing. Strength of recovery was found to be an inverse function of the duration of extinction, suggesting that exposure to nonreinforced stimuli in some manner modified the target memory. The possibility that the extinction period reactivated the memory, which then underwent weakening through further stimulus exposure, was largely ruled out by a subsequent control experiment (Morgan \& Riccio, Experiment 2).

Studies showing that stimulus generalization gradients flatten over time (for a review, see Riccio, Ackil, \& BurchVernon, 1992; Thomas, 1981), a phenomenon widely in- 
terpreted as reflecting the forgetting of stimulus attributes, suggest another strategy for investigating the malleability of an inaccessible memory. This change in the generalization gradient provides a potentially useful marker for assessing the characteristics of inaccessible memory. Presumably, stimulus attributes associated with inaccessible or encapsulated memory should be insensitive to the passage of time and thus would not be influenced by a retention interval. Moreover, in contrast with the extinction manipulation (Morgan \& Riccio, 1994), this approach involves spontaneous change rather than direct experimental intervention. Since one interpretive issue in the extinction study is whether the memory modification occurred at the time of extinction or at the time of testing (see Morgan \& Riccio, 1994, for a discussion), the use of time as the independent variable circumvents this problem.

Of particular relevance to the present experiment is a study by Richardson and Riccio (1986) showing that, when memory was recovered following hypothermia-induced AA, information concerning background/contextual stimuli was also recovered, as indicated by a performance decrement when contextual cues were changed at testing. Richardson and Riccio found that rats that were recooled and tested $24 \mathrm{~h}$ after having been trained while hypothermic displayed recovery from amnesia only when testing occurred with the same external contextual cues as those present during training. Thus, these results indicated that recovered memory contained information about the environmental context in which it was initially coded.

Because recovered memory retains information about contextual stimuli, it should be possible to examine what, if any, changes occur to the contextual generalization gradient of an amnestic memory after a longer retention interval. That is, will an amnestic memory recovered after a long (1-week) interval be independent of a change in the context at testing, or will the recovered memory remain context specific? Either of these two outcomes would be of interest, as the latter would indicate that amnestic memories are somehow encapsulated and protected from processes that normally influence intact memories, whereas the former outcome would suggest that the forgetting of stimulus attributes over time is such a robust process that it influences inaccessible (amnestic) memories.

The appropriateness of the hypothermia-induced amnesia paradigm for this research is demonstrated by evidence of its temperature dependency. Repeated studies (Riccio, Hodges, \& Randall, 1968; Richardson, Guanowsky, Ahlers, \& Riccio, 1984; Richardson, Riccio, \& Morilak, 1983) have shown that induction of hypothermiainduced amnesia requires a reduction of body temperature to a specific threshold level (below $22^{\circ} \mathrm{C}$ ). Reduction of body temperature that does not attain this threshold will not result in any evidence of memory impairment. The fact that a drastic reduction in body temperature $\left(\sim 15^{\circ} \mathrm{C}\right)$ must be attained for amnesia to occur suggests that hypothermia is not simply acting as a disambiguating cue for conditioned fear (see Bouton \& Nelson, 1998). Certainly, body temperature becomes a salient condition for the animal without a full $15^{\circ} \mathrm{C}$ reduction; yet, such subthreshold levels of cooling does not result in memory impairment. Furthermore, recovery of hypothermia-induced amnesia occurs with only a minimal $\left(7-8^{\circ} \mathrm{C}\right)$ reduction in body temperature (Morgan \& Riccio, 1994; Richardson et al., 1984), a finding inconsistent with state-dependent or disambiguation explanations that recommend a recovery change of state equivalent to that originally present during training. Although the neurophysiological changes underlying hypothermia-induced amnesia and recovery have not been determined, repeated demonstrations of the specificity of this cooling threshold strongly suggest the involvement of some temperature-dependent neurological mechanism.

\section{METHOD}

\section{Subjects}

Fifty-six adult (350-500 g) male Sprague-Dawley rats served as subjects. All the animals were experimentally naive at the start of the experiment. The subjects were individually housed and maintained on a 15:9-h light:dark cycle. Food and water were available ad lib.

\section{Apparatus}

Training and testing were conducted in two similarly constructed black-white shuttleboxes (A and B), located in different experimental rooms. Both were constructed of Plexiglas and divided into two equalsized compartments. One compartment, including the lid, was made of black Plexiglas, and the other compartment of white Plexiglas and a clear lid. The floors of both shuttleboxes consisted of 2-mm stainless steel grids, spaced $1 \mathrm{~cm}$ apart. A matched impedance shock source was connected to the grids on the black side of each shuttlebox, to which an unscrambled footshock $(125 \mathrm{~V} / 3 \mathrm{sec})$ could be delivered. Shuttlebox A measured $20 \times 17 \times 17 \mathrm{~cm}$ and had a guillotine door $(10 \times 7)$ separating the two compartments. Shuttlebox B measured $21 \times 19 \times 17 \mathrm{~cm}$ and had a $11 \times 7 \mathrm{~cm}$ guillotine doorway. Shuttlebox A was housed in a room dimly illuminated by a single $15-\mathrm{W}$ incandescent bulb, whereas Shuttlebox B was in a room brightly illuminated by fluorescent lighting. White noise was on at all times in the dimly illuminated room only. The behavior of each subject was monitored visually by means of a tilted mirror under the center of the apparatus.

\section{Procedure}

Following 2 days of 5-min handling, the subjects were randomly divided into seven groups of eight (see Table 1). On Day 1 of the experiment, hypothermia was induced in each rat by immersing it, while in a restraining tube, to the neck in a $2-3^{\circ} \mathrm{C}$ waterbath until its colonic temperature dropped to $21^{\circ} \pm 1^{\circ} \mathrm{C}$ (normal body temperature is approximately $36^{\circ} \mathrm{C}$ ). Typically, rats were retained in the bath for $10 \mathrm{~min}$ to attain the target temperature, but times were adjusted slightly on the basis of weight differences.

Fear conditioning took place once each subject rewarmed to $30^{\circ} \pm 1^{\circ} \mathrm{C}$ (mean interval to rewarm was $90 \mathrm{~min}$ ). The fear conditioning procedure began by placing the rat in the white side of Shuttlebox A, facing away from the closed guillotine door. After $10 \mathrm{sec}$, the guillotine door was opened, and the latency to cross into the black compartment (all four paws) recorded. The door was then lowered and a $3-\sec (125-\mathrm{V})$ inescapable footshock delivered. The subject was then immediately removed from the black compartment and returned to its home cage.

To assess acquisition of the task, the subjects in the immediate test group (ImmTest) were returned to the white (safe) side of the training apparatus $5 \mathrm{~min}$ after training, where they underwent a 10 -min passiveavoidance retention test. During testing, no footshocks were delivered, but two measures of retention were recorded: latency to enter the black compartment for the first time and the total time spent on the white (safe) side (TTW) during the 10 -min test session. High-latency and TTW scores were indicative of good retention. 
Table 1

Experimental Design

\begin{tabular}{llll}
\multicolumn{1}{c}{ Group } & \multicolumn{1}{c}{ Day 1 (Training) } & \multicolumn{2}{c}{ Test Interval } \\
\hline ImmTest & Train (in A) at $30^{\circ} \mathrm{C} /$ Test in A & & 7 Days \\
1-Hypo/Same & Train (in A) at $30^{\circ} \mathrm{C}$ & Recool $\rightarrow$ Test in A & \\
1-Hypo/Diff & Train (in A) at $30^{\circ} \mathrm{C}$ & Recool $\rightarrow$ Test in B & \\
1-Norm/Same & Train (in A) at $30^{\circ} \mathrm{C}$ & Test in A & Recool $\rightarrow$ Test in A \\
7-Hypo/Same & Train (in A) at $30^{\circ} \mathrm{C}$ & & Recool $\rightarrow$ Test in B \\
7- Hypo/Diff & Train (in A) at $30^{\circ} \mathrm{C}$ & & Test in A \\
7-Norm/Same & Train (in A) at $30^{\circ} \mathrm{C}$ & &
\end{tabular}

On Day 2 (24 h after training) groups designated 1-Hypo/Same and 1-Hypo/Diff received a second hypothermia treatment in order to induce recovery of the training memory. As in previous work reversing hypothermia-induced AA (Morgan \& Riccio, 1994; Richardson et al., 1984), the subjects were recooled to $24^{\circ} \pm 1^{\circ} \mathrm{C}$. Once their colonic temperature returned to $30^{\circ} \pm 1^{\circ} \mathrm{C}$, they were given a 10 -min passive avoidance retention test in either the same (Same) or different (Diff) context from training. A group designated 1-Norm/Same was also tested $24 \mathrm{~h}$ after training; however, this group was not recooled, but was tested in the original training context. This group served as a control to indicate the extent of AA $24 \mathrm{~h}$ after training. Initial crossover latencies and TTW scores were recorded for each of the four groups tested.

The remaining three groups were not tested until 1 week after training. At this time, two of the groups (7-Hypo/Same and 7-Hypo/Diff) were recooled and tested in a manner identical to that for the 24-h Hypo groups previously described. Thus, Group 7-Hypo/Same was tested in the original training context, whereas Group 7-Hypo/Diff was tested in the alternative context. The remaining group ( $7 / \mathrm{Same}$ ) was not recooled prior to testing in the original training context, thereby serving as an indicator of the extent of AA following a 1-week delay interval.

\section{Data Analysis}

Analyses were performed on both the latency and the TTW measures throughout the experiment; however, because the outcomes were similar for each measure, generally only the TTW scores will be presented. All parametric analyses were performed using the analysis of variance (ANOVA) procedure from the statistical analysis system (SAS). Nonparametric analyses were calculated, when necessary, as outlined in Howell (1987)

\section{RESULTS}

Initial crossover latencies prior to training were similar for all seven groups [medians (in sec) $=10.5(7-\mathrm{Hypo} /$ Diff), 12.5 (7-Hypo/Same), 14.5 (1-Hypo/Diff), 17.0 (ImmTest), 19.5 (1-Hypo/Same), 23.0 (7-Norm), and 30.5 (1-Norm)]. An ANOVA performed on these initial (training) crossover latencies confirmed this observation $[F(6,49)=1.17, p=.34]$. Thus, prior to training with footshock, step-through performance was similar for all groups.

Retention testing, which took place immediately after fear conditioning for Group ImmTest, resulted in ceiling-level retention scores (median TTW and latency both equaled $600 \mathrm{sec}$ ). A Wilcoxon matched-pairs signedranks analysis comparing training and test scores from these subjects confirmed that test latencies were reliably longer than training latencies $[t(8)=0, p<.004]$. These results established that subjects cooled prior to training did acquire the appropriate conditioned fear response and were capable of retrieving this learning while hypothermic.
Retention testing for the remaining six groups took place either 1 or 7 days following training. Across all six groups, a significant effect of the experimental treatments was detected $[F(5,42)=3.78, p<.006$, for TTW; $F(5,42)$ $=4.29, p<.003$, for latency]. Subsequent pairwise comparisons, using linear contrasts, revealed that the current procedures were effective in producing AA and that reexposure to hypothermia prior to testing alleviated this AA (see Figure 1). Furthermore, a similar pattern of amnesia and recovery was observed whether retention testing took place 1 day after training (1-Hypo/Same vs. 1-Norm/ Same, $t=2.42, p<.02$ ) or 7 days after training ( $7-\mathrm{Hypo}$ Same vs. 7-Norm/Same, $t=2.04, p<.05$ ).

The results of greatest interest were those yielded by comparisons of the Hypo/Same and Hypo/Diff groups at each retention interval ( 1 or 7 days). Figure 1 suggests, and statistical analyses confirmed, that performance that was significantly disrupted by a shift in contextual cues after 1 day was no longer impaired when this shift was delayed until 7 days after training. At the 1 -day interval, recooled subjects tested in the same context as that in which they were trained (1-Hypo/Same) performed significantly better than recooled subjects tested in an environmental context different from that at training (1-Hypo/Diff; $t=$ $3.74, p<.001$ ). Further evidence of sensitivity to contextual stimuli was demonstrated by the lack of difference between group 1-Hypo/Diff and the amnestic subjects in group 1-Norm/Same, which were not recooled $(t=1.31$, $p=.20$ ). However, this sensitivity to a shift in contextual cues was not maintained during the 7-day retention interval, as is demonstrated by the similar test performance of Groups 7-Hypo/Same and 7-Hypo/Diff $(t=0.60, p=$ $.55)$. It should be noted that this lack of difference between the Same and the Diff groups after a 1 week delay was not the result of degraded performance in the 7-Hypo/Same group since Groups 1-Hypo/Same and 7-Hypo/Same also did not differ $(t=1.41, p=.17)$. Thus, the shift in contextual cues resulted in a disruption in performance for the 1 day delay group but not the 7-day delay group.

Further evidence for a change in memory having taken place over the 1-week interval is offered by the finding of a significant interaction between the retention interval and the test context $[F(1,28)=4.30, p=.047]$. An examination of group differences within this interaction term indicated that group 1-Hypo/Diff was impaired. relative to both recooled groups tested in the original training context $(t=3.49, p=.002$ vs. 1 -Hypo/Same; $t=2.18$, 


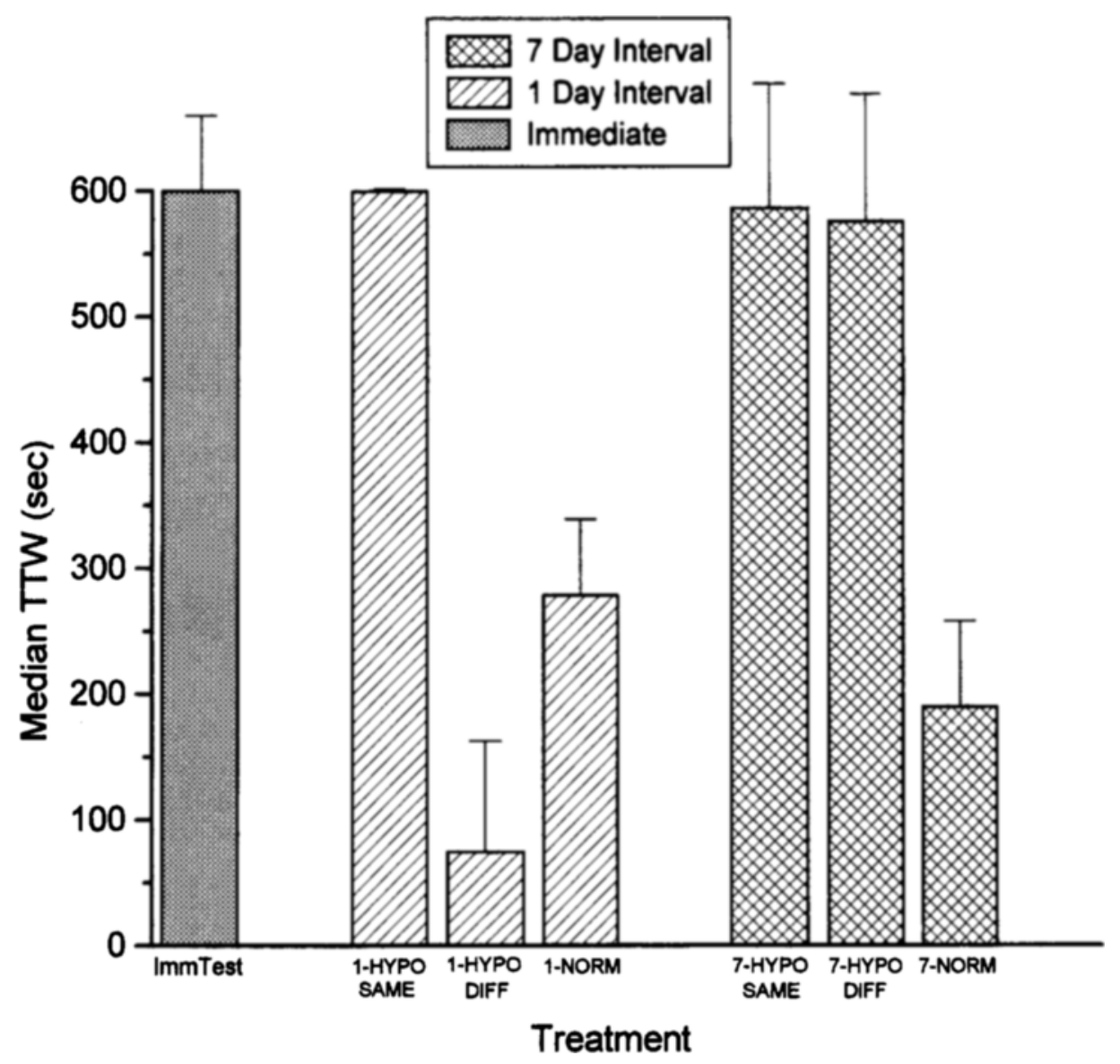

Figure 1. Median total-time-on-white (TTW) scores for subjects tested immediately, 1 day, and 7 days after training. The first term in each two-term group designation indicates the subject's temperature at testing, and the second term indicates whether the subjects were tested in the environmental context in which they had been trained (Same) or not (Diff). Error bars depict the standard error of the mean.

$p=.038$ vs. 7 -Hypo/Same), but only approached significance when compared to group 7-Hypo/Diff $(t=1.62$, $p=.117)$. As in the earlier comparisons, Groups 7-Hypo/ Same and 7-Hypo/Diff both displayed equally strong fear $(t=0.56, p=.58)$, demonstrating the loss of sensitivity to contextual change following the 1 -week interval.

\section{DISCUSSION}

The present experiment examined whether a loss of precise memory for stimulus details, commonly observed in experiments investigating the effects of delayed testing on intact memory, would occur when a memory is inaccessible. By using a reversible amnesia, we were able to test for any changes in memory for attributes. The data suggest two interesting conclusions: (1) Memory recovery was context specific when testing was conducted $24 \mathrm{~h}$ after training, a finding that replicated previous results by Richardson and Riccio (1986), and (2) this context specificity of recovery was not maintained when recooling and testing were delayed for 1 week. The flattening of the contextual generalization gradient in amnestic animals replicates an earlier preliminary and unpublished study in our laboratory (Meehan, Gordon, \& Nardecchia, 1989).

The finding that the very same change in context that impaired memory recovery $24 \mathrm{~h}$ after training had no effect on performance after 1 week implies that memory of the contextual cues present during training did not remain stable during the 1-week delay interval, despite the fact that the amnesia remained strong throughout this time. Furthermore, the substantial memory recovery demonstrated by the subjects tested in the original training context 1 week after training suggests that certain attributes of the training memory were forgotten more rapidly than others. In particular, specific information about the contextual stimuli was forgotten over a 1 -week interval, whereas information regarding the actual conditioned fear response remained intact, a finding reminiscent of previous context shift studies with intact memories (W. R. McAllister \& D. E. McAllister, 1963; Perkins \& Weyant, 1958; for reviews, see Riccio, Rabinowitz, \& Axelrod, 1994, and Riccio, Richardson, \& Ebner, 1984).

An alternative interpretation for this differential rate of forgetting between the context and the conditioned fear response could be made on the basis of the accessibility of these attributes during the amnestic period. Although it was possible to measure the accessibility of the target fear information during the amnestic period, in the presence of amnesia it was not possible to directly assess memory for the context. Thus, a possible argument could be made that memory for the context was not inaccessible. However, this alternative interpretation is unlikely for a number of reasons: (1) There is no reason to assume that the contextual cues and the target response are separate, distinct memories. (2) One would expect an active contextual memory to produce some residual fear when testing took place in the normal state. However, this did not happen, as is demonstrated by both TTW and latency scores (the latter not depicted). (3) The context was not sufficient to cue memory of fear conditioning at normal body temperature. Although a direct test for contextual memory is not possible during amnesia, the lack of residual fear 
and the inability of contextual stimuli to cue the fear response indirectly suggest that memory for the entire configuration of training events was inaccessible while rats were maintained at normal body temperature.

A basic issue in animal memory research concerns the extent to which information must be active in order to be modified. For example substantial evidence indicates that the experimental induction of amnesia depends not on the age of the target memory but on its being in an active state. This activity can be achieved either because the information was recently acquired (i.e., temporally graded retrograde amnesia) or through cuing and reactivation of an old memory (Mactutus, Ferek, George, \& Riccio, 1982; Mactutus, Riccio, \& Ferek, 1979; Misanin, Miller, \& Lewis, 1968; Robbins \& Meyer, 1970; Schneider \& Sherman, 1968). Thus, retrograde amnesia declines as the interval between learning and amnestic treatment increases but can be induced beyond the temporal gradient if subjects are exposed to the training cues prior to administration of the amnestic agent (see Lewis, 1979, for a review). However, the present findings, in conjunction with those reported for extinction (Morgan \& Riccio, 1994), suggest that more mundane sources of forgetting do not require memory to be active, or even accessible, to achieve their effects. Using a very different paradigm to examine contextual updating of memory in human infants, Boller and Rovee-Collier have recently reached a similar conclusion: "a reactivated memory need not have become accessible (i.e., returned to working memory) in order to be modified" (1994, p. 254).

As is true for many other memory malleability paradigms, determining whether the introduction of a new memory alters (erases or updates) the target information or competes with it at the time of testing is problematic (Loftus \& Hoffman, 1989; McCloskey \& Zaragoza, 1985). Is the original memory actually modified, or do conflicting memories coreside? Although Morgan and Riccio (1994) proposed that extinction ex posure acted on the inaccessible fear memory at the time of the manipulation, they acknowledged that their design could not rule out an alternative, more parsimonious interpretation: Two independent memories were established (CS associated with shock, CS associated with no shock) that then competed for expression at the time of testing. The present finding that the contextual gradient flattens over time in amnesic animals would seem to cast doubt on an interpretation that focuses on processes at testing. The forgetting of attributes almost surely depends on processes occurring over time, but this is precisely when the target information is inaccessible. Furthermore, because no active manipulation was used to modify memory, it appears rather unlikely that the establishment of conflicting memories and subsequent competition between them can serve as a satisfactory explanation for the present findings.

To the extent that the malleability of inaccessible memory proves to be a general principle, it raises questions concerning the accuracy of repressed (or suppressed) memories that are later recovered (reactivated) The present findings, taken together with those reported earlier (Morgan $\&$ Riccio, 1994), suggest that inaccessible memories are not necessarily isolated or protected from the influences that modify intact information. More generally, these paradigms may prove useful in developing an experimental model for examining issues related to inaccessible memories.

\section{REFERENCES}

BELLI, R. F. (1989). Influences of misleading postevent information: Misinformation, interference, and acceptance. Journal of Experimental Psychology: General, 118, 72-85.

BOLLER, K., \& RoveE-COLLIER, C. (1994). Contexted updating of infant's reactivated memories. Developmental Psychobiology, 27, 241-256.

Bouton, M. E., \& Nelson, J. B. (1998). The role of context in classical conditioning: Some implications for cognitive behavior therapy. In W. O'Donohue (Ed.), Learning and behavior therapy (pp. 59-84). Boston: Allyn \& Bacon.

ERDELYI, M. H. (1996). The recovery of unconscious memories. Chicago: University of Chicago Press.

Gordon, W. C., McCracken, K. M., Dess-Beech, N., \& Mowrer, R. R. (1981). Mechanisms for the cueing phenomenon: The addition of the cueing context to the training memory. Learning \& Motivation, 12, 196-211

HowelL, D. C. (1987). Statistical methods in psychology. Boston: PWS-Kent.
LEwIS, D. J. (1979). Psychobiology of active and inactive memory. $P_{S y-}$ chological Bulletin, 86, 1054-1083.

LiNDSAY, D. S. (1990). Misleading suggestions can impair eyewitness' ability to remember event details. Journal of Experimental Psychology: Learning, Memory, \& Cognition, 16, 1077-1083.

LofTUS, E. F. (1993). The reality of repressed memories. American Psychologist, 48, 518-537.

LOFTUS, E. F., \& HoFFMAN, H. G. (1989). Misinformation and memory: The creation of new memories. Journal of Experimental Psychology: General, 118, 100-104.

Mactutus, C. F., Ferek, J. M., George, C. A.. \& Riccio, D. C. (1982). Hypothermia-induced amnesia for newly acquired and old reactivated memories: Commonalities and distinctions. Physiological Psychology, 10, 79-95.

Mactutus, C. F., Riccio, D. C., \& Ferek, J. M. (1979). Retrograde amnesia for old (reactivated) memory: Some anomalous characteristics. Science, 204, 1319-1320.

MCAllister, W. R., \& MCAllister, D. E. (1963). Increase over time in the stimulus generalization of acquired fear. Journal of Experimental Psychology, 65, 576-582.

MCCloskey, M., \& Zaragoza, M. (1985). Misleading postevent information and memory for events: Arguments and evidence against memory impairment hypotheses. Journal of Experimental Psychology: General, 114, 1-16.

Meehan, S. M., Gordon, T., \& Nardecchia, D. G. (1989, May). Memory for stimulus attributes following recovery from amnesia $24 \mathrm{hrs}$ or 7 days after training. Paper presented at the annual meeting of the Midwestern Psychological Association, Chicago.

Misanin, J. R., Miller, R. R., \& Lewis, D. J. (1968). Retrograde amnesia produced by electroconvulsive shock after reactivation of a consolidated memory trace. Science, 160, 554-555.

Morgan, R. E., \& Riccio, D. C. (1994). Extinction of an amnestic memory in rats: Evidence for the malleability of "inaccessible" information. Learning \& Motivation, 25, 431-446.

Perkins, C. C., \& Weyant, R. G. (1958). The interval between training and test trials as determiner of the slope of generalization gradients. Journal of Comparative \& Physiological Psychology, 51, 596-600.

Riccio, D. C., ACKIL, J. K., \& BURCh-Vernon, A. (1992). Forgetting of stimulus attributes: Methodological implications for assessing associative phenomena. Psychological Bulletin, 112, 433-445.

Riccio, D. C., Hodges, L. A., \& Randall, P. K. (1968). Retrograde amnesia produced by hypothermia in rats. Journal of Comparative \& Physiological Psychology, 66, 618-622.

Riccio, D. C., Rabinowitz, V. C., \& Axelrod, S. (1994). Memory: When less is more. American Psychologist, 49, 917-926.

Riccio, D. C., Richardson, R., \& Ebner, D. L. (1984). Memory retrieval deficits based upon altered contextual cues. Psychological Bulletin, 96, 152-165.

Richardson, R., Guanowsky, V., Ahlers, S. T., \& Riccio, D. C. (1984). Role of body temperature in the onset of, and recovery from, hypothermia-induced anterograde amnesia. Phisiological Psychology, 12, 125-132

Richardson, R., \& Riccio, D. C. (1986). An examination of a contex tual component of memory following recovery from anterograde amnesia in rats. Physiological Psychology, 14, 75-81.

Richardson, R., Riccio, D. C., \& Morilak, D. (1983). Anterograde memory loss induced by hypothermia in rats. Behavioral \& Neural Biology, 37, 76-88.

Richardson, R., Riccio, D. C., \& SMollfer, D. (1987). Counterconditioning of memory in rats. Animal Learning \& Behavior, 15, 321-326.

RobBins, M. J., \& Meyer, D. (1970). Motivational control of retrograde amnesia. Journal of Experimental Psychology, 84, 220-225.

Schneider, A. M., \& Sherman, W. (1968). Amnesia: A function of the temporal relation of footshock to electroconvulsive shock. Science. 159, 219-221.

Thomas, D. R. (1981). Studies of long-term memory in the pigeon. In N. E. Spear \& R. R. Miller (Eds.), Information processing in animals Memory mechanisms (pp. 257-290). Hillsdale. NJ: Erlbaum.

(Manuscript received May 9. 1997: revision accepted for publication January 2, 1998.) 